\title{
The Short- and Long-term Causal Relationships Between Self-compassion, Trait Mindfulness, Caregiver Stress, and Depressive Symptoms in Family Caregivers of Patients with Lung Cancer
}

\author{
Chia-Chen Hsieh ${ }^{1}$ (D) Zhong-Zhe Lin ${ }^{2} \cdot$ Chao-Chi Ho ${ }^{3}$. Chong-Jen $\mathrm{Yu}^{4,5} \cdot$ Hsiu-Jung Chen ${ }^{6} \cdot$ Yu-Wen Chen ${ }^{7}$ (1) \\ Fei-Hsiu Hsiao ${ }^{1,8}$
}

Accepted: 28 April 2021 / Published online: 5 May 2021

(c) The Author(s), under exclusive licence to Springer Science+Business Media, LLC, part of Springer Nature 2021

\begin{abstract}
Objectives Using a prospective longitudinal design, this paper examines a serial mediation model of the associations between self-compassion, trait mindfulness, caregiver stress, and depressive symptoms among the family caregivers of patients with lung cancer.

Methods A four-wave design was used, with initial assessment (T1) and three follow-ups, at the $2^{\text {nd }}$ month (T2), the $5^{\text {th }}$ month (T3), and the $8^{\text {th }}$ month (T4). A total of 123 family caregivers completed the baseline measurements, including caregiver stress, self-compassion, trait mindfulness, and depressive symptoms. Data were analyzed by serial mediation models to determine the causal ordering of these variables.

Results Nearly one-quarter of the family caregivers suffered from clinically significant depressive symptoms and the severity of their depression remained unchanged throughout the 8-month follow-up period. Both cross-sectional and longitudinal path analyses revealed that the relationship between self-compassion and depressive symptoms was mediated sequentially by trait mindfulness and caregiver stress. The subscale analysis indicated that the association of higher compassionate action with fewer depressive symptoms was through chain-mediating effects of higher mindful awareness and lower caregiver stress.

Conclusions Family caregivers who have higher levels of self-compassion tend to have more mindfulness; greater mindfulness leads to lower levels of perceived caregiving stress which, in turn, links to fewer symptoms of depression. Both selfcompassion and mindfulness could be regarded as protective factors for caregivers to reduce caregiving stress and depression.
\end{abstract}

Keywords Trait mindfulness $\cdot$ Self-compassion $\cdot$ Caregiver stress $\cdot$ Depressive symptoms $\cdot$ Caregiver $\cdot$ Lung cancer

Depressive symptoms are commonly experienced by caregivers of cancer patients (Geng et al., 2018). Studies have found that $22-60 \%$ of family caregivers of lung cancer patients suffer from clinical depression (Nightingale et al., 2019; Siminoff et al., 2010). Lung cancer patients' heavy symptom burdens

Fei-Hsiu Hsiao

hsiaofei@ntu.edu.tw

1 School of Nursing, College of Medicine, National Taiwan University, Taipei, Taiwan

2 Department of Medical Oncology, National Taiwan University Cancer Center, Taipei, Taiwan

3 Department of Internal Medicine, National Taiwan University Hospital, Taipei, Taiwan

4 National Taiwan University Hospital Hsinchu Branch, Hsinchu, Taiwan and low survival rates lead their family members to be in a high-risk group for suffering from depressive symptoms and psychological distress (Nipp et al., 2016; Tan et al., 2018).

Depression in caregivers is mainly caused by the stress that arises from providing care for their ill family members

5 College of Medicine, National Taiwan University, Taipei, Taiwan

6 Department of Educational Psychology and Counseling, National Taiwan Normal University, Taipei, Taiwan

7 Department of Social Work, National Taiwan University, Taipei, Taiwan

8 Department of Nursing, National Taiwan University Hospital, Taipei, Taiwan 
(Geng et al., 2018; Milbury et al., 2013). However, in addition to caregiving burdens, the family caregivers of lung cancer patients also commonly experience uncertainty regarding the patients' disease prognosis (Mosher et al., 2013). It has been found that caregiving burdens generally either remain unchanged or increase over a 6-month period (Grant et al., 2013; Milbury et al., 2013). While undertaking long-term caregiving burdens, family caregivers commonly experience physical and emotional exhaustion, which is referred to as compassion fatigue (Lynch et al., 2018). Their depressive symptoms and psychological distress influence their capacity to provide compassionate care to their loved ones (Day \& Anderson, 2011).

Finlay-Jones's (2017) narrative review proposes a mechanism for the relationships between self-compassion, mindfulness, stress, and depression, in an adaptive emotion regulation framework. Neff (2003) conceptualizes selfcompassion as an individual's capacity for non-judgmental awareness of inner experiences (mindfulness), for acknowledging that suffering is part of the human condition (common humanity), and for responding in a self-soothing and supportive (self-kindness) way to difficult events. According to Gilbert et al.'s (2017) theory, which takes an evolutionary motivational approach, self-compassion consists of two competencies in emotional regulation: engagement and action. Self-compassionate engagement describes the motives to engage, to be aware, to emotionally connect, to empathize, to tolerate, and to make sense of suffering. Selfcompassionate action, alternatively, involves a person being able to shift attention and thinking, to adapt contentmentfocused actions in a soothing system that elicits feelings such as safety and warmth. Neff (2003) and Gilbert et al. (2017) both consider trait self-compassion to be developed through secure attachment, which facilitates an individual's capacity for kindness towards the self.

In Finlay-Jones's (2017) study, self-compassion is consistently shown to be negatively associated with depressive symptoms. By integrating the cross-sectional and experimental quantitative literature with Neff's (2003) and Gilbert et al.'s (2017) models of self-compassion, Finlay-Jones (2017) demonstrates that the impact of selfcompassion on positive emotional regulation stems from its positive influence on the cognitive reappraisal of stress, toward an adaptive view of stress, emotional acceptance and tolerance. There is also a probability that self-compassion is associated with a decrease in the use of negative coping strategies, such as rumination, self-criticism, emotional suppression, and avoidance (Finlay-Jones, 2017). Drawing from path analyses of cross-sectional data, Subic-Wrana et al. (2014) suggest that the non-judgmental awareness of inner experience and self-kindness in selfcompassion is associated with an increase of awareness of emotional experience, which is regarded as a foundation for the further use of positive coping strategies, such as cognitive reappraisal.

Cross-lagged panel analyses show that self-compassion predicted depressive symptoms and the occurrence of a major depressive episode over time (Krieger et al., 2016). Recent studies in both clinical and nonclinical settings have consistently identified self-compassion to be negatively associated with depressive symptoms and maladaptive emotion regulation strategies, and particularly those of rumination and avoidance (Bakker et al., 2019; Inwood \& Ferrari, 2018; Zhu et al., 2019). Xu et al.'s (2020) recent study also found that self-compassion moderated the impact of caregiver burden on depressive symptoms among Chinese caregivers of cancer patients. These results support the notion that self-compassion plays a role as a buffer against causes of depressive symptoms, such as the negative cognitive appraisal of stress, self-focused rumination and criticism, emotional suppression, and avoidance. Nevertheless, more studies implementing a longitudinal design are required for the demonstration of the potential path between selfcompassion and depressive symptoms in the caregivers of cancer patients.

Mindfulness is defined as the psychological state of paying attention and focusing on the present moment with nonjudgment (Kabat-Zinn, 1994). Baer et al. (2006) describe mindfulness as consisting of five aspects: observation, description, acting with awareness, acceptance without judgment, and non-reactivity. Jaffray et al. (2016) conducted a systematic review of quantitative and qualitative studies that revealed that enhancements in the capacity for mindfulness, through mindfulness-based intervention, are associated with fewer depressive symptoms and caregiver burden, and greater quality of life. In a further systematic review (Alsubaie et al., 2017) of 18 clinical trials, which investigated mechanisms of change in mindfulness-based intervention, it was identified that the main mediators of reducing depressive symptoms were increased mindfulness and self-compassion, and decreased rumination, worry, and emotional reactivity.

Evidence from neurobiological studies indicates that cultivating a greater capacity for mindfulness may decrease automatic emotional responding and, therefore, also reduce depressive symptoms (Paul et al., 2013). Mindfulness decreases the unrealistic appraisal of stressful events because it leads individuals to shift attention from a ruminative to a present-centered focus, encouraging acceptance towards the experience of a present moment rather than avoidance of that experience (Shapiro et al., 2006). Mindfulness, thus, creates for the individual opportunities for reflection, which enable more adaptive and flexible ways of responding to difficult situations (Grecucci et al., 2015; Shapiro et al., 2006).

However, barriers have been found to the practice of mindfulness-based intervention for the general population. 
A later disengagement in mindfulness intervention was predicted for individuals with baseline levels of worry and rumination (Banerjee et al., 2018). A qualitative study explores the experiences of healthcare staff engaging in a mindfulness-based intervention (Banerjee et al., 2017). Staff difficulties in engaging in mindfulness were related to problems tolerating negative thoughts and being self-critical. However, a cross-sectional study utilizing mediation analysis indicates that self-compassion increases the individual's motivation to engage in mindfulness training (Rowe et al., 2016). This result is suggestive of the potential role that self-compassion may play in facilitating higher levels of mindfulness. Bluth and Blanton (2014) made a similar finding, using a cross-sectional path analysis, of a reciprocal relationship between mindfulness and self-compassion, in regulating emotional wellbeing. However, the association between self-compassion and mindfulness is not well-tested in studies with a longitudinal design.

While both self-compassion and mindfulness can buffer the impacts of stress on depressive symptoms, self-compassion leads to a probable increase in mindfulness. This is because being more compassionate toward the self may enable the individual both to be less self-critical and, relatedly, to better engage with their inner emotional experiences in stressful situations. This study, therefore, adopts a prospective longitudinal design to test a sequential mediation hypothesis: higher levels of self-compassion are related to greater trait mindfulness (first-order mediator), less caregiving stress (second-order mediator), and reductions in depressive symptoms. According to this hypothesis, selfcompassion engagement and action subscales are associated with five facets of mindfulness. To confirm the relationship between self-compassion and mindfulness, an alternative hypothesis of trait mindfulness at T1 predicting self-compassion at $\mathrm{T} 2$ was also examined.

\section{Methods}

\section{Participants}

A longitudinal and prospective survey was conducted from January 2017 to February 2019. Participants were recruited via the outpatient department of oncology at a medical teaching hospital. Patients with non-small cell lung cancer identified the main family members who provided them with physical or psychological support. These family members were invited to participate in the study. Participants were excluded if they were younger than 20 years of age.

A total of 123 family caregivers (69 females and 54 males) participated in the study. The mean age of participants was 50.6 years $(\mathrm{SD}=12.17$, range $=23-74)$. Most participants were married (78.86\%), employed (63.41\%), religious (78.86\%), and had undergone education at least to a college level of education $(62.60 \%)$. Over half of the caregivers were the spouses or partners of patients (64.23\%). Most caregivers (91.06\%) lived with the patients. The mean length of time for being a caregiver was approximately 34 months (range $=0-204$ ). Participant characteristics are shown in Table 1.

A total of 123 family caregivers completed the first assessment (T1). During the follow-up periods, a total of 55 participants left the study before its completion: 43 at $\mathrm{T} 2,5$ at T3, and 7 at T4. A total of 13 (24\%) participants left the study due to the death or worsening conditions of their ill family member. The other reasons for participants not attending follow-up appointments were a lack of time to participate in the study or a loss of contact.

\section{Procedure}

A four-wave design was used, with initial assessment (T1) and three follow-ups after T1: at the $2^{\text {nd }}$ month (T2), $5^{\text {th }}$ month (T3), and $8^{\text {th }}$ month (T4). After making an appointment with each family caregiver, trained interviewers explained to the participants the study's aims, its procedure, and its potential ethical issues, before they gave their informed consent to participate in the study. All participants were then given a packet of questionnaires and were asked to return the first questionnaire within a fortnight, using the pre-stamped envelope provided. At each follow-up time, the participants were contacted by a research assistant to request the next questionnaire to be returned.

\section{Measures}

Depressive Symptoms Family caregivers' depressive symptoms were assessed using the 21-item Beck Depression Inventory-II (BDI-II) to measure the presence and severity of depressive symptoms (Beck et al., 1996). Respondents responded to each item on a 4-point, Likert-type scale, from 0 ("not at all") to 3 ("extreme form of each symptom"). The total scores ranged from 0 to 63 , with higher scores indicating greater depressive symptoms. Internal consistency reliability estimates for the BDI-II in the current sample were held with a high degree of confidence $(\alpha=0.93)$.

Caregiver Stress The Kingston Caregiver Stress Scale (KCSS) assesses family caregivers' perceptions of their levels of stress (Sadak et al., 2017). This 10-item scale measures different sources of stress: caregiving ( 7 items), family ( 2 items), and financial issues (1-item). Their stress levels were reported on a 5-point rating scale, from 1 ("feeling fine/ no stress") to 5 ("extreme stress"). The total scores range from 10 to 50. Higher scores represent higher levels of caregiver stress. Internal consistency reliability estimates for 
Table 1 Participant characteristics

\begin{tabular}{|c|c|c|c|c|}
\hline & \multirow{2}{*}{$\begin{array}{l}\text { Cross-sectional } \\
\text { Baseline }(n=123)\end{array}$} & \multicolumn{3}{|l|}{ Longitudinal follow-up } \\
\hline & & Completed group $(n=68)$ & $\begin{array}{l}\text { Incomplete } \\
\text { group }(n=55)\end{array}$ & $p$ value \\
\hline Age (mean/SD), years (rang 23-74) & $50.60(12.17)$ & $52.72(10.28)$ & $47.98(13.82)$ & 0.037 \\
\hline Gender $(\%)$ & & & & 0.675 \\
\hline Male/female & $43.90 / 56.10$ & $45.59 / 54.41$ & $41.82 / 58.18$ & \\
\hline Education level (\%) & & & & 0.336 \\
\hline High school and below/ undergraduate and above & $37.40 / 62.60$ & $41.18 / 58.82$ & $32.73 / 67.27$ & \\
\hline Marital status (\%) & & & & 0.052 \\
\hline Single cohabitation, $\%$ & $21.14 / 78.86$ & $14.71 / 85.29$ & $29.09 / 70.91$ & \\
\hline Working status (\%) & & & & 0.240 \\
\hline Unemployed / employed & $36.59 / 63.41$ & $41.18 / 58.82$ & $30.91 / 69.09$ & \\
\hline Religion (\%) & & & & 0.868 \\
\hline Without/with & $21.14 / 78.86$ & $20.59 / 79.41$ & $21.82 / 78.18$ & \\
\hline Comorbid medical illness (\%) & & & & 0.433 \\
\hline Without/with & $56.10 / 43.90$ & $52.94 / 47.06$ & $60.00 / 40.00$ & \\
\hline Caregivers' relationship to patient (\%) & & & & 0.245 \\
\hline Partner & 64.23 & 72.06 & 54.55 & \\
\hline Child & 28.46 & 22.06 & 36.36 & \\
\hline Parent & 4.07 & 2.94 & 5.45 & \\
\hline Sibling & 3.25 & 2.94 & 3.64 & \\
\hline Living with patient (\%) & & & & 0.559 \\
\hline Without/with & $8.94 / 91.06$ & $10.29 / 89.71$ & $7.27 / 92.73$ & \\
\hline Length of time as caregiver (in months) (mean/SD) (range 0-204) & $33.58 / 33.71$ & $33.44 / 39.83$ & $33.75 / 24.47$ & 0.960 \\
\hline Hours of providing daily care (mean/SD) (range $0-24)$ & $8.63(8.16)$ & $9.21 / 8.38$ & $7.90 / 7.91$ & 0.377 \\
\hline
\end{tabular}

the KCSS in the current sample were also held with a high degree of confidence $(\alpha=0.93)$.

Trait Mindfulness For the purposes of the study, mindfulness was regarded as a personality trait and was assessed by using the 39-item Five Facet Mindfulness Questionnaire (FFMQ) (Baer et al., 2006). FFMQ consisted of five subscales to assess mindful observation, description, awareness, non-judgment, and non-reactivity. Participants rated each item, from 1 ("never or very rarely true") to 5 ("very often or always true"). Higher scores on the FFMQ indicate greater levels of trait mindfulness. The FFMQ showed acceptable internal consistency reliability in the current sample: $\alpha=0.73$ to 0.85 .

Self-Compassion The 13-item self-compassion scale measured persons' abilities to have compassion toward themselves (Gilbert et al., 2017). This scale consists of two subscales, to measure two dimensions: engagement and action. The compassionate engagement subscale assessed one's motivation to care for self-wellbeing, acknowledge and display sensitivity to suffering, sympathy, distress tolerance, empathy, and acceptance. An example of compassionate engagement is depicted in the following statement: "I notice and am sensitive to my distressed feelings when they arise in me." The compassionate action subscale assessed an individual's ability to acknowledge, learn about, and act on what is beneficial in alleviating distress. An example of a statement depicting compassionate action is: "I think about and come up with helpful ways to cope with my distress." Participants were asked to respond on a 10-point Likert point scale, from 1 ("Never") to 10 ("Always"). The results for these two subscales were then combined to give a total score, with higher scores indicating greater levels of selfcompassion. The internal consistency for this step in the study was acceptable $(\alpha=0.79)$.

Demographic Information Information was collection regarding each participant's age, gender, education level, marital status, working status, religions, relationship to the patient, and caregiving conditions.

\section{Data Analyses}

Two types of data were missing from the study. "Item missing data" refers to items in a questionnaire being incomplete. "Wave missing data" refers to questionnaires that were not provided by participants at follow-up intervals. For item 
missing data, there were two missing items at baseline and three missing items at follow-up intervals. Missing value analysis (in SPSS) revealed that these values were missing completely at random, allowing for imputation using the estimation-maximization method. $T$-tests and chi-square tests were used to examine whether participants in the baseline assessment differed from those who completed all assessments. Sensitivity analyses were also conducted to assess whether incomplete cases influenced the results of the longitudinal mediation model. A multiple imputation in SPSS was conducted, generating five imputed datasets. There were no significant differences in the results between analyses using the imputed data and the original data. Based on sensitivity analysis, the exclusion of incomplete cases from our analyses did not, then, appear to influence our conclusions. Therefore, for cases in which participants did not complete all questionnaires, missing data in the follow-up periods were excluded from the analysis.

For cross-sectional data, the relationships between study variables were assessed with a two-tailed Pearson correlation coefficient $(r)$. Multiple linear regression analyses were used to determine predictors of depressive symptoms. The serial mediation analyses were used to examine the association of self-compassion with depressive symptoms through serial mediators (trait mindfulness and caregiving stress). The serial mediation models were analyzed using the Model 6 of PROCESS macro for SPSS (Hayes, 2017). As recommended by Hayes (2017), the bootstrapping methods (with 5000 bootstrap samples) were used to estimate indirect effects. The indirect effect is significant if zero does not fall within the 95\% bootstrapped confidence intervals (95\% CI). Path coefficients, in an unstandardized form, were used to represent the strength of the relationship between study variables. The assumed order of the serial pathway was as follows. T1 selfcompassion (independent variable) would increase $\mathrm{T} 1$ trait mindfulness (first mediator). T1 trait mindfulness would, in turn, decrease T1 caregiver stress (second mediator) and, thus, also decrease T1 depressive symptoms (dependent variable) (see Fig. 2). For analyzing subscales, a facet-level analysis examined the association between each subscale of self-compassion and trait mindfulness in the serial mediation models.

For the longitudinal data, the relationships between study variables were explored using linear mixed models. Time points were formatted as time variant, to consider changes over time; the random effect was used to account for intra-subject variability. A compound symmetry covariance matrix-which gave the lowest AIC values-was used to indicate a model effect within a subject error correlation structure. The longitudinal mediation models examining the temporal precedence between the study variables were analyzed using the serial mediation approach (Hayes PROCESS Model 6). This process first involved examining whether changes in the scales of mindfulness and self-compassion occurred over time.
The findings showed that there were no significant changes in these scales, suggesting that individual values remained significantly "invariable" over time. We then examined how self-compassion, trait mindfulness, and caregiver stress were related to depressive symptoms. The baseline (T1) self-compassion scores were entered as the independent variable. The trait mindfulness scores at time 2 (T2) were entered as the first mediator. The caregiver stress at time 3 (T3) was entered as the second mediator. Finally, scores for depressive symptoms at time 4 (T4) were entered as the dependent variable. To confirm the relationship between self-compassion and mindfulness, an alternative model in which trait mindfulness at $\mathrm{T} 1$ and selfcompassion at $\mathrm{T} 2$ was also examined.

\section{Results}

\section{Preliminary Analyses}

The analysis of the differences between participants who completed all questionnaires at follow-up intervals and those who did not indicated that there was only one significant difference, which was in terms of age. The sub-sample of family caregivers who completed all four assessments were older than those who did not complete the assessments $(M$ $\pm \mathrm{SD}=52.72 \pm 10.28$ versus $47.98 \pm 13.82, p=0.037)$. The descriptive statistics and differences between completed and incomplete groups are presented in Table 1. Self-compassion scores were higher among family caregivers who completed all four assessment waves than those who did not ( $M$ $\pm \mathrm{SD}=71.53 \pm 10.99$ versus $65.87 \pm 14.54, p=0.019$ ). There were no significant differences in mean scores of depressive symptoms, levels of caregiver stress, and trait mindfulness between the two groups.

\section{Trajectory of Caregivers' Depressive Symptoms}

In the initial assessment, $24.39 \%(n=30)$ of family caregivers scored above the BDI-II cut-off point of 13, for clinically significant depressive symptoms (Junior et al., 2019). Figure 1 shows that family caregivers who exhibited higher levels of depressive symptoms at baseline had no significant changes during the 8-month follow-up period.

\section{Cross-sectional Associations Between Self-Compassion, Trait Mindfulness, Caregiver Stress, and Depressive Symptoms}

There were no associations between sociodemographic variables and depressive symptoms $(p>0.05)$. Table 2 shows that levels of self-compassion correlate positively with trait mindfulness and correlated negatively with depressive symptoms but were unrelated to levels of caregiving stress. Trait mindfulness correlated negatively with caregiver stress 


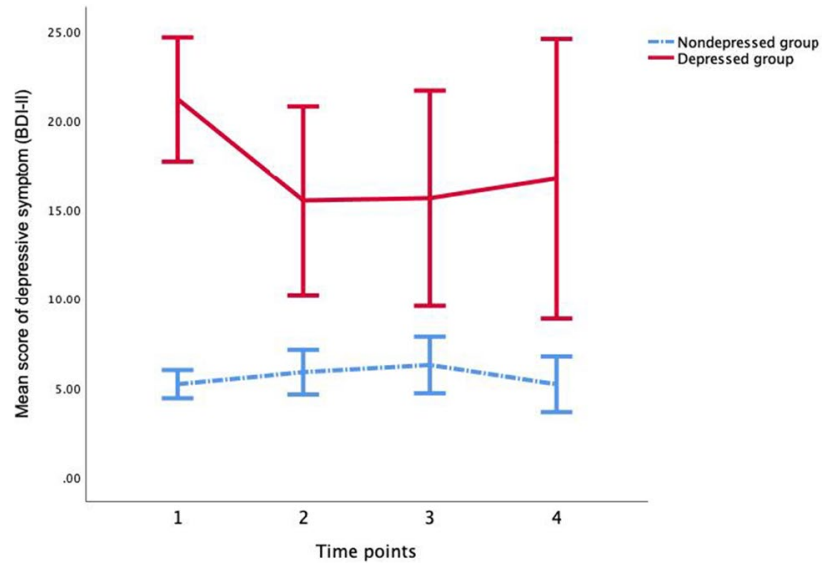

Fig. 1 Mean score of depressive symptoms across time points

and depressive symptoms, while caregiving stress correlated positively with depressive symptoms.

The multiple regression analysis revealed that depressive symptoms significantly correlate with levels of selfcompassion $(b=-0.105, t(119)=-2.050, p=0.043)$, trait mindfulness $(b=-0.183, t(119)=-4.257, p<0.001)$, and caregiver stress $(b=0.551, t(119)=7.403, p<0.001)$. Table 3 indicates that these three factors explained $50 \%$ of the variance in depressive symptoms among participants (adjusted $\left.R^{2}=0.509, F(3,119)=43.16, p<0.001\right)$.

A conceptual diagram of the serial mediation model and path coefficients is presented in Fig. 2a. The indirect effect of testing the path from self-compassion to depressive symptoms through the mediators of trait mindfulness and caregiver stress was significant (effect $=-0.065$, BootSE $=0.028,95 \%$ BootCI $=(-0.128,-0.022))$. According to path coefficients, T1 self-compassion was positively related to $\mathrm{T} 1$ trait mindful awareness $(B=0.617, \mathrm{SE}=0.098$, $p<0.001)$, which in turn was inversely related to T1 caregiver stress $(B=-0.192, \mathrm{SE}=0.050, p<0.001)$. The lower $\mathrm{T} 1$ caregiver stress value was related to fewer T1 depressive symptoms $(B=0.551, \mathrm{SE}=0.075, p<0.001)$. The serial path revealed that self-compassion was inversely related to depressive symptoms, and this relationship was serially mediated through an increase in trait mindfulness and a decrease in caregiving stress.

Table 2 Descriptive statistics and correlation matrix among study variables at baseline $(n=123)$

\begin{tabular}{lllll}
\hline Variables & Mean $(\mathrm{SD})$ & 1 & 2 & 3 \\
\hline $\begin{array}{l}\text { 1.Depressive symp- } \\
\text { toms }\end{array}$ & $9.11(8.91)$ & 1 & & \\
2.Caregiving stress & $19.62(8.05)$ & $.590^{* * *}$ & & \\
3.Self-compassion & $69.00(12.96)$ & $-.310^{* * *}$ & .012 & \\
4.Mindfulness & $133.12(16.10)$ & $-.546^{* * *}$ & $-.283^{* *}$ & $.496^{* * *}$ \\
\hline
\end{tabular}

${ }^{*} p<0.05 ; * * p<0.01 ; * * * p<0.001$ (2-tailed)
Further analysis was conducted to identify the associations between the two subscales of self-compassion (compassionate engagement and action) and the five subscales of trait mindfulness (observation, description, acting with awareness, non-judgment, or non-reactivity) with caregiver stress and depressive symptoms. When either subscale (of self-compassion or trait mindfulness) was tested the other subscale was included as a covariate in the analysis. At facetlevel analyses, as shown in Fig. 2b, serial mediation analyses revealed a significant indirect effect of compassionate action on depressive symptoms, increasing trait mindfulness awareness, and decreasing caregiver stress (effect $=-0.114$, BootSE $=0.037,95 \%$ BootCI $=(-0.191,-0.050))$.

Compassionate action had a significantly positive effect on trait mindful awareness $(B=0.339, \mathrm{SE}=0.055, p<0.001)$ while trait mindful awareness had a significant negative effect on levels of caregiver stress $(B=-0.779, \mathrm{SE}=0.135$, $p<0.001)$. Finally, decreased caregiver stress was correlated with reductions of depressive symptoms $(B=0.431$, $\mathrm{SE}=0.079, p<0.001)$. These results demonstrate that the path of higher compassionate action as relating to lower depressive symptoms is through the positive impacts of compassionate action on increasing trait mindfulness in the aspect of awareness, and decreasing levels of caregiver stress. There were no significant findings in the compassion engagement domain or other subscales of trait mindfulness in the path analysis.

\section{Longitudinal Associations Between Self-compassion, Trait Mindfulness, Caregiver Stress, and Depressive Symptoms}

The results from the mixed models indicate that both trait mindfulness and caregiver stress had an impact on depressive symptoms (see Table 3). Specifically, when adjusted for time, the higher trait mindfulness levels predicted lower scores of depressive symptoms $(b=-0.180$, $t(342)=-7.234, p<0.001)$. Higher caregiver stress levels predicted higher scores of depressive symptoms $(b=0.387$, $t(341)=7.578, p<0.001)$.

Longitudinal mediation models were used to determine temporal precedence and causal-ordering assumptions among variables over time. As shown in Fig. 2c, the indirect effects of self-compassion at $\mathrm{T} 1$ were significant in decreasing depressive symptoms at $\mathrm{T} 4$ through the mediators of trait mindfulness at $\mathrm{T} 2$ and the management of caregiver stress levels at T3 (effect $=-0.079$, BootSE $=0.041,95 \%$ BootCI $=(-0.171,-0.014))$. There were three sequential relationships in the pathway. T1 self-compassion was positively related to $\mathrm{T} 2$ trait mindful awareness $(B=0.879$, $\mathrm{SE}=0.175, p<0.001) . \mathrm{T} 2$ trait mindfulness awareness was inversely related to $\mathrm{T} 3$ caregiver stress $(B=-0.125$, $\mathrm{SE}=0.053, p=0.022)$. Finally, lower T3 caregiver stress 
Table 3 Cross-sectional and longitudinal analysis of the effects of caregiver stress, selfcompassion, mindfulness on the depressive symptoms

\begin{tabular}{|c|c|c|c|c|c|c|}
\hline & \multicolumn{6}{|c|}{ Depressive symptoms } \\
\hline & \multicolumn{3}{|c|}{ Cross-sectional analysis $^{\text {a }}$} & \multicolumn{3}{|c|}{ Longitudinal analysis ${ }^{b}$} \\
\hline & $b$ & SE & Sig & $b$ & SE & Sig \\
\hline Intercept & 29.832 & 5.492 & $<0.001$ & 26.759 & 3.618 & $<0.001$ \\
\hline Caregiving stress & 0.551 & 0.074 & $<0.001$ & 0.387 & 0.051 & $<0.001$ \\
\hline Self-compassion & -0.105 & 0.051 & 0.043 & -0.018 & 0.029 & 0.539 \\
\hline Mindfulness & -0.183 & 0.043 & $<0.001$ & -0.180 & 0.025 & $<0.001$ \\
\hline
\end{tabular}

${ }^{a}$ Cross-sectional analysis based on linear regression model

${ }^{\mathrm{b}}$ Longitudinal analysis based on linear mixed-effects model was related to fewer T4 depressive symptoms $(B=0.717$, $\mathrm{SE}=0.166, p<0.001)$. These results demonstrate that the negative association of baseline self-compassion with depressive symptoms at the $8^{\text {th }}$ month can be mediated through two serial mediators: an increase of trait mindfulness in the $2^{\text {nd }}$ month and a decrease of caregiver stress in the $5^{\text {th }}$ month. To confirm the relationship between self-compassion and mindfulness, an alternative model was examined for the path from trait mindfulness (T1) to depressive symptoms (T4), through the mediators of self-compassion (T2) and caregiver stress (T3). The results for this path were not significant (effect $=-0.005$, BootSE $=0.018,95 \%$ BootCI $=(-0.033,0.038))$. Finally, the subscale analysis found that there were no significant findings regarding the associations between the specific domains of self-compassion and the facets of trait mindfulness with caregiver stress and depressive symptoms.

\section{Discussion}

This study found that nearly one-quarter of the family caregivers of patients with lung cancer suffered from clinically significant depressive symptoms, and the severity of their depression remained unchanged throughout the 8-month follow-up period. In adopting a longitudinal design, our study identified that increases in self-compassion were associated with less depressive symptoms through the chain-mediating effects of mindfulness and caregiver stress. Moreover, the association of baseline self-compassion with depressive symptoms at the $8^{\text {th }}$ month was mediated sequentially through the impacts of self-compassion on trait mindfulness at the $2^{\text {nd }}$ month and caregiver stress at the $5^{\text {th }}$ month. The path analysis demonstrates that family caregivers who have higher levels of selfcompassion have a propensity for more mindfulness; greater mindfulness leads to lower levels of caregiving stress which, in turn, links to fewer symptoms of depression.

The cross-sectional study showed that higher levels of both self-compassion and mindfulness were associated with lower levels of depression (Lopez et al., 2016). In a previous study that implemented a cross-sectional design, path analyses indicated a reciprocal relationship between self-compassion and trait mindfulness in regulating emotional wellbeing, among a sample of 67 adolescents (Bluth \& Blanton, 2014). However, in our longitudinal analyses, only trait mindfulness remained a significant predictor of depressive symptoms across the 8-month follow-up period. Moreover, our path analysis shows that the relationship between self-compassion and depressive symptoms is indirect, and that their relationship is affected by the chain-mediators of trait mindfulness and caregiver stress. Our results suggest that self-compassion allows caregivers to develop greater mindful awareness of difficult thoughts
Fig. 2 a Cross-sectional mediation model. b Facets of crosssectional mediation model. c Longitudinal mediation model. $\mathrm{T} 1=$ time 1 (baseline), $\mathrm{T} 2=$ time 2 (2-month followup), T3 = time 3 (5-month follow-up), T4 = time 4 (8-month follow-up). All statistics are $\mathrm{b}$ statistics, ${ }^{* * *} p<.001,{ }^{*} p<.05$

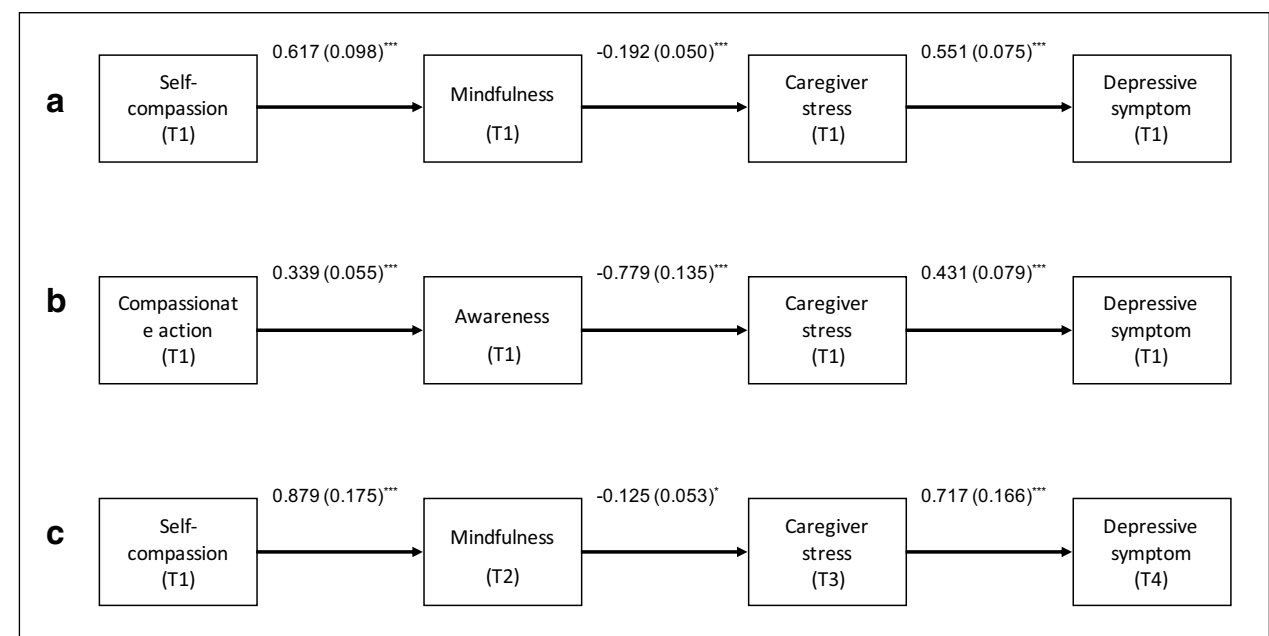


and feelings. People who practice self-compassion have a lower probability of being self-critical and a higher probability of engaging in self-care and positive change, aiding them in being mindful and responding constructively to difficult experiences (Barnard \& Curry, 2011; Jay Miller et al., 2019). Being mindful enables people to be more aware and accepting of their experiences of the present moment, leading to reductions in stress levels and other negative outcomes (Colgan et al., 2019; Creswell \& Lindsay, 2014). Moreover, as supported by previous findings (Pires et al., 2018), people with higher self-compassion scores report greater mindfulness and sensitivity to emotional stimuli.

An individual's difficulties in engaging with mindfulness practices may sometimes be attributable to problems tolerating negative thoughts and a tendency to be self-critical (Banerjee et al., 2018). Thus, developing a capacity for self-compassion allows individuals to be less self-critical which, in turn, increases their ability to engage in mindfulness practices (Rowe et al., 2016). Consistent with the results of Finlay-Jones's (2017) review, our path analysis supports the notion that caregivers' higher levels of selfcompassion facilitate their ability for mindfulness, reducing their perceptions of caregiving stress and decreasing depressive symptoms. In our subscale analysis, a higher score in the domain of self-compassionate action was associated with higher mindful awareness, and by which stress levels and depressive symptoms were then decreased. The ability to perform compassionate actions allows individuals to convert their intentions into effective actions and reduce their suffering (Gilbert et al., 2017). Accordingly, it is conceivable that family caregivers capable of taking compassionate action might more effectively use mindful awareness to respond to stress in an adaptive way to reduce depression when present.

In conclusion, our study identifies a path by which depressive symptoms may decrease. This path begins with self-compassion and is mediated through trait mindfulness and its effects on caregiver stress. The results suggest that assisting caregivers in being more compassionate to self may enable them to become more mindful of their inner experience. Depression interventions for caregivers of cancer patients may, therefore, benefit in targeting their ability to be self-compassionate, and especially their proficiency for compassionate action. Such an approach paves the way for training caregivers in mindfulness practices, as involving attention to present moment experiences without judgment, to reappraise stress in open and constructive ways.

\section{Limitations and Future Research Directions}

This study used Gilbert et al.'s (2017) new measure for selfcompassion, as incorporating both engagement and action domains. Self-compassionate engagement measures six characteristics, in terms of motives the motives to engage, to be aware, to emotionally connect, to empathize, to tolerate, and to make sense of suffering. Self-compassionate action measures competency in adapting contentment-focused actions to alleviate and prevent suffering. Due to the relatively low number of studies using this scale, there is a limitation on the extent to which the findings of the present study are generalizable. Further studies are required to examine and explore these two domains of self-compassion in clinical samples.

A further limitation is related to the fact that most of the caregivers who participated in the study were either the spouse or partner of a lung cancer patient. Consequently, the results and conclusions drawn here may not be as applicable to other kinds of patient-caregiver relationships. However, a main limitation of this study stems from the rate of $44.72 \%$ of participants that did not complete all questionnaires. The differences in age as well as scores for self-compassion, between the participants who did and did not completed the study, should be noted. Younger caregivers, and those who were employed, had a higher probability of not completing a follow-up survey. Conversely, family caregivers who had higher levels of selfcompassion also had tendencies to be more motivated in terms of self-care, which may explain their motivation for completing follow-up activities (Jay Miller et al., 2019). Finally, the research findings may be affected by a common methodological bias, due to the self-reporting measures using multiple-item scales, which might lead to spurious effects due to response styles, social desirability, and priming effects.

Nevertheless, this study identifies a path by which we might demonstrate how short-term and long-term depressive symptoms can be reduced, through the impacts of self-compassion and trait mindfulness on caregivers' levels of stress. Future observational studies may follow this rationale, by investigating hypotheses regarding chains of causal relationships among self-compassion, trait mindfulness, caregiver stress, and depressive symptoms. Of particular interest is the possibility of controlling for confounding factors at each level of such an investigation, in a comprehensive structural model, as is made possible through structural equation modeling (SEM). Future intervention studies may, furthermore, focus on testing the effects of a mindful self-compassion program on reducing depressive symptoms for caregivers of cancer patients, under the hypothesis that the serial mediation effects of a chain process from self-compassion, mindfulness to caregiver stress levels, act to reduce such symptoms.

Authors' Contributions $\mathrm{CCH}$ : designed and executed the study, analyzed the data, and wrote the first draft of the manuscript. ZZL, $\mathrm{CCH}$, and CJY: assisted with participant recruitment. HJC and YWC: provided critical feedback and helped shape the manuscript. FHH: designed and executed the study, collaborated in the writing and editing of the final manuscript. All authors approved the final version of the manuscript for submission. 


\section{Declarations}

Ethics Approval All procedures performed in the studies involving human participants were in accordance with the ethical standards of the institutional and/or national research committee and with the 1964 Helsinki declaration and its later amendments or comparable ethical standards. The protocol for this study has received approval from the Institutional Review Board of the National Taiwan University Hospital.

Informed Consent Informed consent was obtained from all the individual participants included in the study.

Conflict of Interest The authors declared that they have no conflicts of interest.

\section{References}

Alsubaie, M., Abbott, R., Dunn, B., Dickens, C., Keil, T. F., Henley, W., \& Kuyken, W. (2017). Mechanisms of action in mindfulnessbased cognitive therapy (MBCT) and mindfulness-based stress reduction (MBSR) in people with physical and/or psychological conditions: A systematic review. Clinical Psychology Review, 55, 74-91. https://doi.org/10.1016/j.cpr.2017.04.008

Baer, R. A., Smith, G. T., Hopkins, J., Krietemeyer, J., \& Toney, L. (2006). Using self-report assessment methods to explore facets of mindfulness. Assessment, 13(1), 27-45. https://doi.org/10.1177/ 1073191105283504

Bakker, A. M., Cox, D. W., Hubley, A. M., \& Owens, R. L. (2019). Emotion regulation as a mediator of self-compassion and depressive symptoms in recurrent depression. Mindfulness, 10, $1169-1180$

Banerjee, M., Cavanagh, K., \& Strauss, C. (2017). A qualitative study with healthcare staff exploring the facilitators and barriers to engaging in a self-help mindfulness-based intervention. Mindfulness, 8(6), 1653-1664

Banerjee, M., Cavanagh, K., \& Strauss, C. (2018). Barriers to mindfulness: A path analytic model exploring the role of rumination and worry in predicting psychological and physical engagement in an online mindfulness-based intervention. Mindfulness, 9(3), 980-992. https://doi.org/10.1007/s12671-017-0837-4

Barnard, L. K., \& Curry, J. F. (2011). Self-compassion: Conceptualizations, correlates, \& interventions. Review of General Psychology, 15(4), 289-303. https://doi.org/10.1037/a0025754

Beck, A. T., Steer, R. A., Ball, R., \& Ranieri, W. (1996). Comparison of Beck depression inventories -IA and -II in psychiatric outpatients. Journal of Personality Assessment, 67(3), 588-597. https://doi.org/10.1207/s15327752jpa6703_13

Bluth, K., \& Blanton, P. W. (2014). Mindfulness and self-compassion: Exploring pathways to adolescent emotional well-being. Journal of Child and Family Studies, 23(7), 1298-1309. https:// doi.org/10.1007/s10826-013-9830-2

Colgan, D. D., Klee, D., Memmott, T., Proulx, J., \& Oken, B. (2019). Perceived stress mediates the relationship between mindfulness and negative affect variability: A randomized controlled trial among middle-aged to older adults. Stress and Health, 35(1), 89-97. https://doi.org/10.1002/smi.2845

Creswell, J. D., \& Lindsay, E. K. (2014). How does mindfulness training affect health? A mindfulness-stress-buffering account. Current Directions in Psychological Science, 23(6), 401-407

Day, J. R., \& Anderson, R. A. (2011). Compassion fatigue: An application of the concept to informal caregivers of family members with dementia. Nursing Research and Practice, 2011, 1-10. https://doi.org/10.1155/2011/408024

Finlay-Jones, A. L. (2017). The relevance of self-compassion as an intervention target in mood and anxiety disorders: A narrative review based on an emotion regulation framework. Clinical Psychologist, 21, 90-103

Geng, H. M., Chuang, D. M., Yang, F., Yang, Y., Liu, W. M., Liu, L. H., \& Tian, H. M. (2018). Prevalence and determinants of depression in caregivers of cancer patients: A systematic review and meta-analysis. Medicine (Baltimore), 97(39), e11863. https://doi.org/10.1097/MD.0000000000011863

Gilbert, P., Catarino, F., Duarte, C., Matos, M., Kolts, R., Stubbs, J., Ceresatto, L., Duarte, J., Pinto-Gouveia, J., \& Basran, J. (2017). The development of compassionate engagement and action scales for self and others. Journal of Compassionate Health Care, 4(4), 1-24

Grant, M., Sun, V., Fujinami, R., Sidhu, R., Otis-Green, S., Juarez, G., Klein, L., \& Ferrel, B. (2013). Family caregiver burden, skills preparedness, and quality of life in non-small cell lung cancer. Oncoloy Nursing Forum, 40(4), 337-346. https://doi. org/10.1188/13.ONF.337-346

Grecucci, A., Pappaianni, E., Siugzdaite, R., Theuninck, A., \& Job, R. (2015). Mindful emotion regulation: Exploring the neurocognitive mechanisms behind mindfulness. Biomed Research International, 2015, 670724. https://doi.org/10.1155/2015/670724

Hayes, A. F. (2017). Introduction to mediation, moderation, and conditional process analysis. (2nd ed.). Guilford Press.

Inwood, E., \& Ferrari, M. (2018). Mechanisms of change in the relationship between self-compassion, emotion regulation, and mental health: A systematic review. Applied Psychology: Health and Well-Being, 10(2), 215-235

Jaffray, L., Bridgman, H., Stephens, M., \& Skinner, T. (2016). Evaluating the effects of mindfulness-based interventions for informal palliative caregivers: A systematic literature review. Palliative Medicine, 30(2), 117-131. https://doi.org/10.1177/0269216315 600331

Jay Miller, J., Lee, J., Niu, C., Grise Owens, E., \& Bode, M. (2019). Self-compassion as a predictor of self-care: A study of social work clinicians. Clinical Social Work Journal, 47(4), 321-331

Junior, A. R., Liebel, G., de Andrade, A. G., Andrade, L. H., Gorenstein, C., \& Wang, Y. P. (2019). Can gender and age impact on response pattern of depressive symptoms among college students? A differential item functioning analysis. Front in Psychiatry, 10, 50. https://doi.org/10.3389/fpsyt.2019.00050

Kabat-Zinn, J. (1994). Wherever you go, there you are. Hyperion.

Krieger, T., Berger, T., \& Holtforth, M. G. (2016). The relationship of self-compassion and depression: Cross-lagged panel analyses in depressed patients after outpatient therapy. Journal of Affective Disorders, 202, 39-45. https://doi.org/10.1016/j.jad. 2016.05.032

Lopez, A., Sanderman, R., \& Schroevers, M. J. (2016). Mindfulness and self-compassion as unique and common predictors of affect in the general population. Mindfulness, 7(6), 1289-1296. https:// doi.org/10.1007/s12671-016-0568-y

Lynch, S. H., Shuster, G., \& Lobo, M. L. (2018). The family caregiver experience - Examining the positive and negative aspects of compassion satisfaction and compassion fatigue as caregiving outcomes. Aging \& Mental Health, 22(11), 1424-1431. https://doi. org/10.1080/13607863.2017.1364344

Milbury, K., Badr, H., Fossella, F., Pisters, K. M., \& Carmack, C. L. (2013). Longitudinal associations between caregiver burden and patient and spouse distress in couples coping with lung cancer. Supportive Care in Cancer, 21(9), 2371-2379. https://doi.org/10. 1007/s00520-013-1795-6

Mosher, C. E., Jaynes, H. A., Hanna, N., \& Ostroff, J. S. (2013). Distressed family caregivers of lung cancer patients: An examination 
of psychosocial and practical challenges. Supportive Care in Cancer, 21(2), 431-437. https://doi.org/10.1007/s00520-012-1532-6

Neff, K. D. (2003). Self-compassion: An alternative conceptualization of a healthy attitude toward oneself. Self and Identity, 2, 85-101

Nightingale, C. L., Steffen, L. E., Tooze, J. A., Petty, W., Danhauer, S. C., Badr, H., \& Weaver, K. E. (2019). Lung cancer patient and caregiver health vulnerabilities and interest in health promotion interventions: An exploratory study. Global Advances in Health and Medicine, 17(8), 1-11. https://doi.org/10.1177/2164956119 865160

Nipp, R. D., El-Jawahri, A., Fishbein, J. N., Gallagher, E. R., Stagl, J. M., Park, E. R., Jackson, V. A., Piril, W. F., Greer, J. A., \& Temel, J. S. (2016). Factors associated with depression and anxiety symptoms in family caregivers of patients with incurable cancer. Annals of Oncoogyl, 27(8), 1607-1612. https://doi.org/10.1093/annonc/ mdw205

Paul, N. A., Stanton, S. J., Greeson, J. M., Smoski, M. J., \& Wang, L. (2013). Psychological and neural mechanisms of trait mindfulness in reducing depression vulnerability. Social Cognitive and Affective Neuroscience, 8, 56-64

Pires, F. B. C., Lacerda, S. S., Balardin, J. B., Portes, B., Tobo, P. R., Barrichello, C. R. C., Amaro, E., \& Kozasa, E. H. (2018). Self-compassion is associated with less stress and depression and greater attention and brain response to affective stimuli in women managers. BMC Women's Health, 18(1), 195. https://doi.org/10. 1186/s12905-018-0685-y

Rowe, A. C., Shepstone, L., Carnelley, K. B., Cavanagh, K., \& Millings, A. (2016). Attachment security and self-compassion priming increase the likelihood that first-time engagers in mindfulness meditation will continue with mindfulness training. Mindfulness, 7, 642-650. https://doi.org/10.1007/s12671-016-0499-7

Sadak, T., Korpak, A., Wright, J. D., Lee, M. K., Noel, M., Buckwalter, K., \& Borson, S. (2017). Psychometric evaluation of kingston caregiver stress scale. Clinical Gerontologist, 40(4), 268-280. https://doi.org/10.1080/07317115.2017.1313349

Shapiro, S. L., Carlson, L. E., Astin, J. A., \& Freedman, B. (2006). Mechanisms of mindfulness. Journal of Clinical Psychology, 62(3), 373-386

Siminoff, L. A., Wilson-Genderson, M., \& Baker, S., Jr. (2010). Depressive symptoms in lung cancer patients and their family caregivers and the influence of family environment. PsychoOncology, 19(12), 1285-1293. https://doi.org/10.1002/pon.1696

Subic-Wrana, C., Beutel, M. E., Brahler, E., Stobel-Richter, Y., Knebel, A., Lane, R. D., \& Wiltink, J. (2014). How is emotional awareness related to emotion regulation strategies and self-reported negative affect in the general population? PLOS ONE, 9(3), e91846. https:// doi.org/10.1371/journal.pone.0091846

Tan, J. Y., Molassiotis, A., Lloyd-Williams, M., \& Yorke, J. (2018). Burden, emotional distress and quality of life among informal caregivers of lung cancer patients: An exploratory study. European Journal of Cancer Care, 27(1), e12691. https://doi.org/10. $1111 /$ ecc. 12691

Xu, S., Zhang, H., \& Wang, J. (2020). Caregiver burden and depression among Chinese family caregivers: The role of self-compassion. Mindfulness, 11, 1647-1654

Zhu, L., Yao, J., Wang, J., Wu, L., Gao, Y., Xie, J., Liu, A., Ranchor, A. V., \& Schroevers, M. J. (2019). The predictive role of selfcompassion in cancer patients' symptoms of depression, anxiety, and fatigue: A longitudinal study. Psycho-Oncology, 28(9), 1918-1925. https://doi.org/10.1002/pon.5174

Publisher's Note Springer Nature remains neutral with regard to jurisdictional claims in published maps and institutional affiliations. 